\title{
Exosome in disease biology, diagnosis, and therapy
}

\author{
Nobuyoshi Kosaka, Yusuke Yoshioka, Takeshi Katsuda, \\ Makiko Ono and Takahiro Ochiya*
}

Division of Molecular and Cellular Medicine, National Cancer Center Research Institute, Tokyo, Japan

For many decades, tools for cell-cell communication were of great concern in various research fields, not only in basic research, but also in medical research. Although a large number of humoral factors, such as cytokines, chemokines, growth factors, hormones, and so on, have been identified and characterized, few molecular mechanisms of cell-cell communication have been clarified. Recently, small membrane vesicles, called exosomes (also known as extracellular vesicles), have been at the center of attention again. Current vigorous worldwide research found that exosomes play multiple important roles in physiological and pathological phenomena. In this review, we will summarize our current reports showing the essential roles of exosomes in cancer development, the novel utility of exosomes to know the status of the patient, and the potential novel treatment choice for neurological disease.

Rec.9/11/2014, Acc.10/29/2014, pp233-239

\footnotetext{
${ }^{*}$ Correspondence should be addressed to:

Takahiro Ochiya, Division of Molecular and Cellular Medicine, National Cancer Center Research Institute, 1-1, Tsukiji, 5-chome, Chuo-ku, Tokyo 104-0045, Japan. Phone: +81-3-3542-2511(ex4800), Fax: +81-3-3541-2685, E-mail: tochiya@ncc.go.jp
}

Key words exosome, extracellular vesicles, cancer biology, diagnosis, disease treatment

\section{Introduction}

Cell-cell communication is a fundamental biological activity in multicellular organisms, and understanding this is crucial for the development of disease treatment. To date, many humoral factors, which are tools for the cells to communicate with other cells, have been found, and many therapeutic tools have been developed from these findings ${ }^{1-3)}$. From this point of view, clarifying the molecular mechanisms of cell-cell communication might be of great interest in medical research. Recently, a novel communication tool for cells has come back into the limelight. This tool, called the exosome, was first discovered in 1980 's $\mathrm{s}^{4,5)}$ and has been thought of as dust from cells. One of the first discoveries of the exosome's role in cellcell communication was in 1990's $s^{6}$. This was cell-cell communication between immunological cells, and the roles of exosomes in immunological reactions have now been recognized ${ }^{7-10}$; however, their contribution to disease progression and their usages have not yet been studied. Recent studies demonstrated that various types of vesicles 
are secreted from cells. One of that, microvesicles, also known as microparticles or shedding vesicles, represent a heterogeneous population of vesicles with a diameter of 100 to $1000 \mathrm{~nm}$ that are released by budding of the plasma membrane ${ }^{11)}$, although there are few report showing their function in cell-cell communication compared with exosome. In this review, we will briefly summarize our current findings about the roles of exosomes in cancer development ${ }^{12,13)}$. In addition, we will also introduce our current reports about the usage of exosomes for diagnostic ${ }^{14)}$ and therapeutic ${ }^{15)}$ purposes. Finally, we will discuss the future direction of exosome usage in medical situations.

\section{Biological meaning of exosomes in cancer}

Exosomes, also known as extracellular vesicles, are 20to $200-n m$ vesicles secreted from living cells ${ }^{16,17)}$, and they contain various types of proteins, RNAs ${ }^{18)}$ (mRNA, noncoding RNA, and microRNAs (miRNAs)), and in some cases, $D_{N A}{ }^{19,20)}$. Exosomes are positive for tetraspanin family proteins, such as CD63, CD81, and CD9 ${ }^{17,21,22)}$, and contain cell surface proteins and cytoplasmic proteins. Their source cells decide the variations of these contents. One of the unique features of exosomes is that these vesicles are able to transfer between their source cells (donor cells) and their target cells (recipient cells). After the discovery of exosomes in immunological cell-cell communication, the biological importance of exosomes became more and more obvious.

It is well known that cancer cells actively communicate with their microenvironmental cells to improve their situation $^{23)}$. For instance, a massive volume of cells leads to a hypoxia status; thus, the cancer cells need oxygen for their survival. To improve the conditions for their survival, cancer cells secrete angiogenic factors, such as FGF-2 and VEGF, or induce their production from microenvironmental cells, and then endothelial cells become proliferative and construct vascular systems in the tumors ${ }^{24)}$. Thus, understanding these humoral factors is direct way to develop cancer therapy, and indeed, some of the current cancer drugs have been developed from the list of humoral factors. From this aspect, we decided to investigate the function of the exosome, which is another humoral factor, in cancer development. Previously, we found that the regulation of exosomal miRNA secretion is controlled by neutral sphingomyelinase 2 (nSMase2), which regulates the biosynthesis of ceramide, and these secretory miRNAs are transferable and functional in recipient cells ${ }^{25)}$. From this observation, we established the metastatic cancer cell lines, whose expression of nSMase2 was abolished or over-produced, to understand whether the modulation of exosome production in malignant cancer cells could be affected by exosomes. The results showed that the modulation of nSMase 2 in malignant cancer cells clearly regulates the metastasis event. Furthermore, we also found that miRNA in exosomes from cancer cells promotes angiogenesis in the tumor and induces metastasis. From this report, we insist that exosomes from cancer cells promote the cancer cell metastasis through controlling their microenvironmental cells, resulting in the enhancement of metastasis ${ }^{12)}$. This conclusion is supported by other groups. For instance, exosomes from melanoma cells mediated the conditioning of lymph nodes and defined microanatomic responses that license the metastasis of melanoma cells ${ }^{26)}$. In addition, exosomes from melanoma cells increased the metastatic behavior of primary tumors by permanently "educating" bone marrow progenitors through the receptor tyrosine kinase $\mathrm{MET}^{27)}$.

Recently, we found a novel exosome-mediated phenomenon in cancer recurrence ${ }^{12}$. Cancer recurrence often occurs, and this is a serious problem for cancer cure. Breast cancer patients often develop metastatic disease years after resection of the primary tumor. The patients are asymptomatic because the disseminated cells appear to become dormant and are undetectable. Further, because recurrence is often more aggressive and untreatable, it is important to identify the mechanisms that enable therapeutic subversion and regrowth. Bone marrow is a common homing tissue for disseminated tumor cells. It is thought that micrometastases form in the bone marrow and then recirculate to invade other, distant organs ${ }^{28)}$. We currently found that exosomes from bone marrow-derived mesenchymal stem cells (MSCs) can be transferred to the bone marrow-metastatic human breast cancer cell line, and it caused the bone marrow-metastatic human breast cancer cell line to be dormant phenotypes. Interestingly, we also clarified that miRNA in exosomes from bone marrowderived MSCs suppresses the expression of MARCKS, which encodes a protein that promotes cell cycling and motility, in the bone marrow-metastatic human breast cancer cell line.

Currently, it is known that exosomes secreted by cancer cells play an important role in cancer progression by promoting angiogenesis ${ }^{19)}$, neutrophil infiltration ${ }^{29)}$, and the education of bone marrow-derived cells ${ }^{27}$. Thus it is an 
attractive idea that targeting the exosomes in cancer cells might be an alternative choice for cancer treatment.

\section{Exosomes in body fluid can be used to monitor human status}

In addition to the roles of exosomes reported by our group and other groups, exosomes contribute much toward pathological phenomena, including cancer, immunological disease $\mathrm{e}^{30,31)}$, neurological disease ${ }^{32}$, and so on. From this point of view, it is natural to think that exosomes can be used to detect these kinds of diseases as well as for diagnosis or trace markers. Indeed, it has been reported that circulating exosomes in cancer patients increased along with the stage of cancer $^{33,34)}$. In addition, the expressions of proteins on the surface of the exosome are higher in an advanced stage of cancer than in non-malignant cancer ${ }^{27)}$. Although there is much evidence showing the existence of exosomes in human body fluids, and this fact will have an outstanding impact against future personal medicine, detecting the exosome in human body fluid remains one of many consuming issues to be solved. One of the most serious problems is that we don't have superior techniques to isolate exosomes with a small amount of human body fluid, and this makes the detection method very difficult.

\section{Detecting exosomes by a novel develop- ing method}

As described earlier, detection of exosomes requires a significant volume of human body fluid, and this is not suitable for the future usage of exosomes in the treatment and/or diagnosis of disease or to monitor the daily health of individuals. For these reasons, many researchers worldwide have attempted to develop highly sensitive and highly specific methods of analyzing exosomes. Conventional methods require a large volume of human body fluid to isolate exosomes and are processed via time-consuming immunoblotting or enzyme-linked immunosorbent assays; these methods are impractical in most clinical settings.

In our laboratory, to realize the usage of exosomes in a clinical situation, we established a highly sensitive and rapid analytical technique for profiling surface proteins in exosomes from human body fluids without an exosome purification step ${ }^{14)}$. This method is simple to use, meaning that many researchers can easily use our techniques without any barriers. The novel screening system we developed, called ExoScreen, is based on an amplified luminescent proximity homogeneous assay using photosensitizer ${ }^{35)}$. Streptavidin-coated donor beads, which capture an analyte-specific biotinylated antibody, and acceptor beads conjugated to a second antibody, which recognizes an epitope of the analyte, were used. The donor beads are excited by $680-\mathrm{nm}$ lasers, resulting in the release of singlet oxygen, which excites an amplified fluorescent signal in the acceptor beads. As a result, the acceptor beads emit light at $615 \mathrm{~nm}$, but only if they are within 200 $\mathrm{nm}$ of the analyte captured by both antibodies. Based on this principal, the detection of larger vesicles, such as apoptotic bodies, shedding vesicles, or protein complexes, is prevented because the average size of exosomes is approximately $100 \mathrm{~nm}$. Further, antigen monomers cannot be detected by this method. One of the big benefits of this assay is that we only need $5 \mu$ of cancer patients' serum to detect circulating cancer-derived exosomes.

To perform the cancer-specific detection of exosomes in human body fluid, cancer-specific antigens in exosomes need to be discovered. Based on the hypothesis that tumorderived exosomes may differ from circulating exosomes in normal physiological conditions, cancer-specific antigens in exosomes can be discovered by comparison of cancerderived exosomes and normal cell-derived exosomes. Thus, protein profiles of exosomes from a colorectal cancer cell line and a colon fibroblast were compared by proteomic analysis, and the amount of CD147, which is the immunoglobulin superfamily member, was found to be significantly higher in the exosomes from cancer cell lines than in those from normal cells. Based on this fact, cancer-derived exosomes in human clinical samples were evaluated by CD147 and CD9 using ExoScreen, and we found that CD147 and CD9 double-positive exosomes were significantly higher in the serum from cancer patients than in that from healthy donors. Most importantly, the amount of CD147 in cancer patient sera reduced afterward, suggesting that the CD147-harboring exosomes originated from cancer cells in the patient. Recently, various techniques to detect exosomes in human body fluids have been developed $^{36,37)}$. Many of them are based on targeting the antigens on exosomes; thus, it is essential to identify the cancer-specific or disease-specific exosome in human body fluid. The current organization of the exosome database might be helpful for this ${ }^{38)}$.

\section{Exosomes as a novel therapeutic tool to cure neurological disease}

As shown above, the exosome itself has a variety of 
functions to mediate the communication between cells. In other words, if we use these exosome functions, we can send the "correcting message" to recipient cells, such as insufficient molecules in sick recipient cells. For instance, we recently revealed that exosome-harbored neprilysin (neutral endopeptidase: NEP) can decrease the $\beta$-amyloid peptide whose accumulation is thought to be one cause of Alzheimer's disease $(A D)^{15}$. NEP is a type II membraneassociated metalloendopeptidase, and it appears to be the most important among several proteases involved in the proteolysis of $A \beta^{39,40)}$, because decreased expression and activity of NEP in AD patients were reported ${ }^{41}$. We know that the expression of neprilysin in adipose tissue-derived MSCs (ADSCs) is higher than that in bone marrow-derived MSCs (BMSCs); thus, we checked the secretion level of the exosome from each MSC. The results showed that the secretion level of the neprilysin-bound exosome is higher in exosomes from ADSCs than those from BMSCs. ADSCs have a great benefit for order-made therapeutic approach using patients' own exosomes, because they are routinely isolated from the stromal vascular fraction of homogenized adipose tissue. This report shows that exosomes can be novel therapeutic tools for the disease.

\section{Conclusion and future perspectives}

In this review, we summarize our recent progress in the basic research and medical research in human disease. Current research in this field aggressively endeavors to quickly reveal many amazing facts about exosomes. Unlike the first discovery of exosomes in 1980's, which thought of exosomes as garbage of the cells, current research indicates that exosomes might be central mediators of cell-cell communication. Thus, many of the sick cells produce abnormal exosomes, such as immunological cells, neurological cells, and cancer cells. From this view, these abnormal exosomes can be used as biomarkers for disease, and we can use exosomes to treat the disease. These facts appear promising for future personal medicine; however, there are still issues to be resolved for medical usage.

Below, we summarize the recent issues to be solved:

1. The biology in every type of disease mediated by exosomes must be clarified in more detail. Currently, much of the knowledge about exosomes is limited to particular research fields, such as cancer, immunology, and neurology. It is possible that the cause of many other diseases is abnormal exosomes from those sick cells. Thus, clarifying these mechanisms will be a great

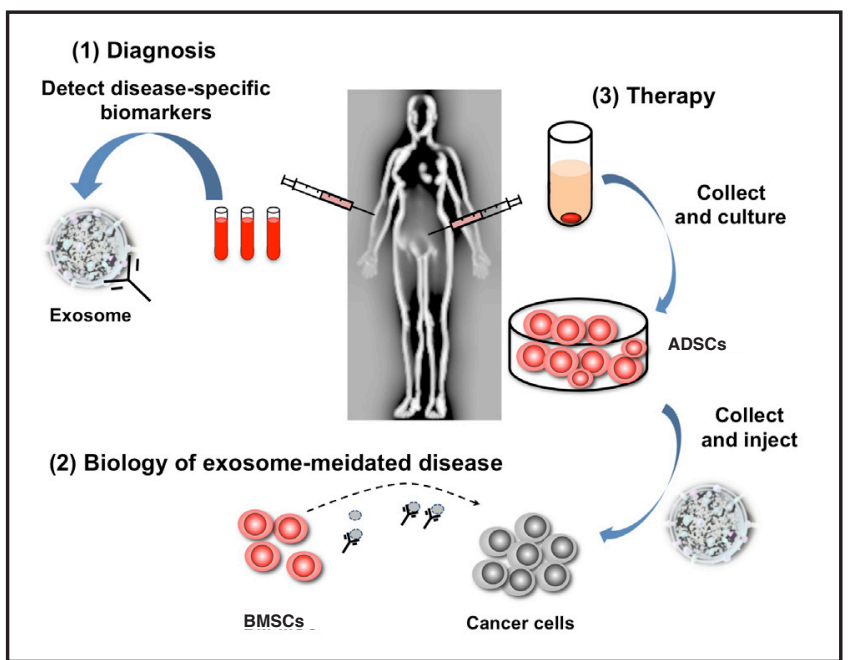

Fig.1 Schematic representation of the proposed strategy for clinical application of exosomes

As shown in this paper, exosome can be used in diagnosis and therapeutic application. For diagnosis, since exosome carries the protein of their originated cells, it is possible to detect disease-specific proteins in non-invasive way, such as Exoscreen (1). On the other hands, if we understand the function of exosome from cancer cells correctly, we can design the strategy for disease treatment, such as capturing exosome by antibody (2). Finally, exosome itself has a huge capacity for disease treatment. To do this, isolating exosome from cells that originate from patients themselves to avoid immuno-reaction caused by foreign substances. In this context, adipose tissue derived mesenchymal stem cells are ideal source for collecting exosome. These exosome can be used not only for cancer treatment but also for disease treatment based on the character of exosome, such as Alzheimer disease (3).

help for further understanding of the diseases.

2. Disease-specific surface markers on exosomes should be listed. Current novel detection methods are based on the method of using antibodies. These methods, of course, are highly specific; however, in this case, we need to know what kinds of surface proteins exist on the exosomes from sick cells. Current ISEV (International Society for Extracellular Vesicles) works on the organizing exosome protein database might be a great help for this.

3. Understanding the detailed character of exosomes, such as half-life, tropism to specific cells, and so on, is essential for further usage of disease treatment by exosome.

Much of the research has been done in the past several years; however, we need to continue in order to understand exosome function and character in more detail. Then we can freely use exosomes in clinical situation (Fig. 1). 


\section{Acknowledgements and Source of Funding}

This work was supported in part by a Grant-in-Aid for the ThirdTerm Comprehensive 10-Year Strategy for Cancer Control, a Grantin-Aid for Scientific Research on Priority Areas Cancer from the Ministry of Education, Culture, Sports, Science and Technology, and the Program for Promotion of Fundamental Studies in Health Sciences of the National Institute of Biomedical Innovation (NiBio), and the Japan Society for the Promotion of Science (JSPS) through the 'Funding Program for World-Leading Innovative R\&D on Science and Technology (FIRST Program)' initiated by the Council for Science and Technology Policy (CSTP), and a Grant-inaid for Project for Development of Innovative Research on Cancer Therapeutics (P-Direct), and Grant-in-Aid for Scientific Research on Innovative Areas ('functional machinery for non-coding RNAs') from the Japanese Ministry of Education, Culture, Sports, Science, and Technology, and Comprehensive Research and Development of a Surgical Instrument for Early Detection and Rapid Curing of Cancer Project (P10003) of the New Energy and IndustrialTechnology Development Organization (NEDO), a research program of the Project for Development of Innovative Research on Cancer Therapeutics (P-Direct), Ministry of Education, Culture, Sports, Science and Technology of Japan.

\section{Conflict of interests}

No potential conflicts of interest were disclosed.

\section{References}

1) Melero-Martin JM, Dudley AC: Concise review: Vascular stem cells and tumor angiogenesis. Stem Cells. 2011; 29: $163-168$

2) Hall K, Ran S: Regulation of tumor angiogenesis by the local environment. Front Biosci. 2010; 15: 195-212.

3) Stevenson CE, Nagahashi M, Ramachandran S, Yamada A, Bear HD, Takabe K: Bevacizumab and breast cancer: what does the future hold? Future Oncol. 2012; 8: 403-414.

4) Pan BT, Johnstone RM: Fate of the transferrin receptor during maturation of sheep reticulocytes in vitro: selective externalization of the receptor. Cell. 1983; 33: 967-978.

5) Johnstone RM, Adam M, Hammond JR, Orr L, Turbide C: Vesicle formation during reticulocyte maturation. Association of plasma membrane activities with released vesicles (exosomes). J Biol Chem. 1987; 262: 9412-9420.

6) Raposo G, Nijman HW, Stoorvogel W, Liejendekker R, Harding CV, Melief CJ, Geuze HJ: B lymphocytes secrete antigen-presenting vesicles. J Exp Med. 1996;
183: 1161-1172.

7)Zitvogel L, Regnault A, Lozier A, Wolfers J, Flament C, Tenza D, Ricciardi-Castagnoli P, Raposo G, Amigorena

$S$ : Eradication of established murine tumors using a novel cell-free vaccine: dendritic cell-derived exosomes. Nat Med. 1998; 4: 594-600.

8)Wolfers J, Lozier A, Raposo G, Regnault A, Théry C, Masurier C, Flament C, Pouzieux S, Faure F, Tursz T, Angevin E, Amigorena S, Zitvogel L. Tumor-derived exosomes are a source of shared tumor rejection antigens for CTL cross-priming. Nat Med. 2001; 7, 297-303.

9)Okoye IS, Coomes SM, Pelly VS, Czieso S, Papayannopoulos V, Tolmachova T, Seabra MC, Wilson MS: MicroRNA-containing T-regulatory-cellderived exosomes suppress pathogenic $\mathrm{T}$ helper 1 cells. Immunity. 2014; 41: 89-103.

10) Robbins PD, Morelli AE: Regulation of immune responses by extracellular vesicles. Nat Rev Immunol. 2014; 14: 195-208.

11) Muralidharan-Chari V, Clancy JW, Sedgwick A, D'Souza-Schorey C: Microvesicles: mediators of extracellular communication during cancer progression. J Cell Sci. 2010; 123: 1603-1611.

12) Kosaka N, Iguchi $H$, Hagiwara K, Yoshioka $Y$, Takeshita F, Ochiya T: Neutral sphingomyelinase 2 (nSMase2)dependent exosomal transfer of angiogenic microRNAs regulate cancer cell metastasis. J Biol Chem. 2013; 288: 10849-10859.

13) Ono M, Kosaka N, Tominaga N, Yoshioka Y, Takeshita F, Takahashi RU, Yoshida M, Tsuda H, Tamura K, Ochiya $\mathrm{T}$ : Exosomes from bone marrow mesenchymal stem cells contain a microRNA that promotes dormancy in metastatic breast cancer cells. Sci Signal. 2014; 7: ra63.

14) Yoshioka $Y$, Kosaka N, Konishi $Y$, Ohta H, Okamoto $H$, Sonoda $H$, Nonaka $R$, Yamamoto $H$, Ishii $H$, Mori M, Furuta K, Nakajima T, Hayashi H, Sugisaki H, Higashimoto H, Kato T, Takeshita F, Ochiya T: Ultrasensitive liquid biopsy of circulating extracellular vesicles using ExoScreen. Nat Commun. 2014; 5: 3591.

15)Katsuda T, Tsuchiya R, Kosaka N, Yoshioka Y, Takagaki K, Oki K, Takeshita F, Sakai Y, Kuroda M, Ochiya T: Human adipose tissue-derived mesenchymal stem cells secrete functional neprilysin-bound exosomes. Sci Rep. 2013; 3: 1197.

16)Raposo G, Stoorvogel W: Extracellular vesicles: 
exosomes, microvesicles, and friends. J Cell Biol. 2013; 200: 373-383.

17) Mathivanan S, Simpson RJ: ExoCarta: A compendium of exosomal proteins and RNA. Proteomics. 2009; 9: 4997-5000.

18) Valadi H, Ekström K, Bossios A, Sjöstrand M, Lee JJ, Lötvall JO: Exosome-mediated transfer of mRNAs and microRNAs is a novel mechanism of genetic exchange between cells. Nat Cell Biol. 2007; 9: 654-659.

19) Ronquist KG, Ronquist G, Carlsson L, Larsson A. Human prostasomes contain chromosomal DNA. Prostate. 2009; 69: 737-743.

20)Balaj L, Lessard R, Dai L, Cho YJ, Pomeroy SL, Breakefield XO, Skog J: Tumour microvesicles contain retrotransposon elements and amplified oncogene sequences. Nat Commun. 2011; 2: 180.

21) Yoshioka Y, Konishi Y, Kosaka N, Katsuda T, Kato T, Ochiya T: Comparative marker analysis of extracellular vesicles in different human cancer types. J Extracell Vesicles. 2013; doi: 10.3402/jev.v2i0.20424.

22) Choi DS, Park JO, Jang SC, Yoon YJ, Jung JW, Choi DY, Kim JW, Kang JS, Park J, Hwang D, Lee KH, Park SH, Kim YK, Desiderio DM, Kim KP, Gho YS: Proteomic analysis of microvesicles derived from human colorectal cancer ascites. Proteomics. 2011; 11: 2745-2751.

23) Hanahan D, Weinberg RA: Hallmarks of cancer: the next generation. Cell. 2011; 144: 646-674.

24) Weis SM, Cheresh DA: Tumor angiogenesis: molecular pathways and therapeutic targets. Nat Med. 2011; 17, 1359-1370.

25) Kosaka N, Iguchi H, Yoshioka Y, Takeshita F, Matsuki Y, Ochiya T: Secretory mechanisms and intercellular transfer of microRNAs in living cells. J Biol Chem. 2010; 285: 17442-17452.

26) Hood JL, San RS, Wickline SA: Exosomes released by melanoma cells prepare sentinel lymph nodes for tumor metastasis. Cancer Res. 2011; 71: 3792-3801.

27) Peinado H, Alečković M, Lavotshkin S, Matei I, CostaSilva B, Moreno-Bueno G, Hergueta-Redondo M, Williams C, García-Santos G, Ghajar C, NitadoriHoshino A, Hoffman C, Badal K, Garcia BA, Callahan MK, Yuan J, Martins VR, Skog J, Kaplan RN, Brady MS, Wolchok JD, Chapman PB, Kang Y, Bromberg J, Lyden D: Melanoma exosomes educate bone marrow progenitor cells toward a pro-metastatic phenotype through MET. Nat Med. 2012; 18: 883-891.

28) Pantel K, Alix-Panabières C, Riethdorf S: Cancer micrometastases. Nat Rev Clin Oncol. 2009; 6: 339351.

29) Bobrie A, Krumeich S, Reyal F, Recchi C, Moita LF, Seabra MC, Ostrowski M, Théry C: Rab27a supports exosome-dependent and -independent mechanisms that modify the tumor microenvironment and can promote tumor progression. Cancer Res. 2012; 72: 4920-4930.

30)Zhang HG, Zhuang X, Sun D, Liu Y, Xiang X, Grizzle WE: Exosomes and immune surveillance of neoplastic lesions: a review. Biotech Histochem. 2012; 87: 161168.

31)Hosseini HM, Fooladi AA, Nourani MR, Ghanezadeh F: The role of exosomes in infectious diseases. Inflamm Allergy Drug Targets. 2013; 12, 29-37.

32)Schneider A, Simons M. Exosomes: vesicular carriers for intercellular communication in neurodegenerative disorders. Cell Tissue Res. 2013; 352, 33-47.

33) Taylor DD, Gercel-Taylor C: MicroRNA signatures of tumor-derived exosomes as diagnostic biomarkers of ovarian cancer. Gynecol Oncol. 2008; 110: 13-21.

34)Gercel-Taylor C, Atay S, Tullis RH, Kesimer M, Taylor DD: Nanoparticle analysis of circulating cell-derived vesicles in ovarian cancer patients. Anal Biochem. 2012; 428: 44-53.

35) Ullman EF, Kirakossian H, Singh S, Wu ZP, Irvin BR, Pease JS, Switchenko AC, Irvine JD, Dafforn A, Skold CN, et al: Luminescent oxygen channeling immunoassay: measurement of particle binding kinetics by chemiluminescence. Proc Natl Acad Sci U S A. 1994; 91: 5426-5430.

36) Shao H, Chung J, Balaj L, Charest A, Bigner DD, Carter BS, Hochberg FH, Breakefield XO, Weissleder R, Lee $\mathrm{H}$ : Protein typing of circulating microvesicles allows realtime monitoring of glioblastoma therapy. Nat Med. 2012; 18: $1835-1840$.

37) Im H, Shao H, Park YI, Peterson VM, Castro CM, Weissleder R, Lee $\mathrm{H}$ : Label-free detection and molecular profiling of exosomes with a nano-plasmonic sensor. Nat Biotechnol. 2014; 32: 490-495.

38) Vesiclepedia: http://microvesicles.org

39) Iwata N, Tsubuki S, Takaki Y, Shirotani K, Lu B, Gerard NP, Gerard C, Hama E, Lee HJ, Saido TC: Metabolic regulation of brain Abeta by neprilysin. Science. 2001; 292: 1550-1552.

40) Iwata N, Tsubuki S, Takaki Y, Watanabe K, Sekiguchi M, Hosoki E, Kawashima-Morishima M, Lee HJ, Hama 
E, Sekine-Aizawa Y, Saido TC: Identification of the major Abeta1-42-degrading catabolic pathway in brain parenchyma: suppression leads to biochemical and pathological deposition. Nat Med. 2000; 6, 143-150.
41)Yasojima K, Akiyama H, McGeer EG, McGeer PL: Reduced neprilysin in high plaque areas of Alzheimer brain: a possible relationship to deficient degradation of beta-amyloid peptide. Neurosci Lett. 2001; 297: 97-100. 